\title{
HOW MUCH BLAME CAN BE PLACED ON LASER PHOTOCOAGULATION FOR FAILURE TO ATTAIN DRIVING STANDARDS?
}

\author{
S. W. MACKIE ${ }^{1}$, L. A. WEBB ${ }^{2}$, B. M. HUTCHISON ${ }^{2}$, H. M. HAMMER ${ }^{2}$, \\ T. BARRIE ${ }^{2}$ and G. WALSH ${ }^{1}$ \\ Glasgow
}

\begin{abstract}
SUMMARY
One hundred consecutive patients who underwent bilateral pan-retinal photocoagulation (PRP) for proliferative diabetic retinopathy were assessed in accordance with the UK Driver and Vehicle Licensing Agency (DVLA) guidelines. Visual acuity was documented, and visual fields were assessed using the Esterman test. Among the $30 \%$ of patients who failed to reach the visual standards required for a driving licence, three groups were identified: those who failed to attain either the required binocular visual acuity $(n=4)$, or visual fields $(n=9)$, or both $(n=17)$. Previous studies reveal a large variation in DVLA field test failure following PRP treatment for proliferative diabetic retinopathy. Our results show a $19 \%$ failure rate solely attributable to treatment, which is at the lower end of previously reported studies (20-80\%). The reasons for this discrepancy are discussed. We conclude that modern treatment procedures for proliferative diabetic retinopathy may be undertaken with the knowledge that in the majority of cases a patient's driving licence is unlikely to be revoked.
\end{abstract}

Laser pan-retinal photocoagulation (PRP) is now established as the treatment of choice for patients with proliferative diabetic retinopathy and reduces the risk of severe visual loss by $50 \%$ or more. ${ }^{1}$ Additional treatment is administered for persistent new vessels until total or near total regression occurs. This allows the retention of central vision in the majority of patients. Argon laser photocoagulation is, however, associated with several complications and side-effects including: angle closure, ${ }^{2}$ exudative retinal detachment, ${ }^{3}$ iatrogenic choroidal neovascu-

From: ${ }^{1}$ Glasgow Caledonian University; ${ }^{2}$ Glasgow Eye Infirmary, Glasgow, UK.

Correspondence to: Mr S. W. Mackie, BSc, MBCO, MFDO, Department of Vision Sciences, Glasgow Caledonian University, Cowcaddens Road, Glasgow G4 0BA, UK. larisation, ${ }^{4}$ macular oedema, ${ }^{5}$ optic neuropathy, ${ }^{6}$ non-progressive lenticular opacities, ${ }^{7}$ constricted visual field ${ }^{8}$ defective hue discrimination, ${ }^{9}$ increased glare ${ }^{10}$ and reduced contrast sensitivity. ${ }^{11}$

Following laser treatment each UK patient is obliged to inform the Driver and Vehicle Licensing Agency (DVLA), ${ }^{12}$ which in turn requests a formal acuity and field test from the patient's medical attendant to assess fitness to drive. DVLA recommendations include:

1. The ability to read a number plate, letter height 3.125 inches, at 67 feet. This geometrically converts to a binocular Snellen visual acuity (VA) of approximately $6 / 15$, but has been shown under realistic conditions to more closely approximate $6 / 10$ using a $50 \%$ pass criterion. ${ }^{13}$

2. A minimal visual field of at least $120^{\circ}$ along the horizontal and of at least $20^{\circ}$ above and below the horizontal measured by perimetry using a $3 \mathrm{~mm}$ white test object at $\frac{1}{3} \mathrm{~m}$ (or equivalent perimetry). ${ }^{14}$

The standard does not define the use of one eye only or both eyes together, since either condition is allowed.

We have investigated a cohort of diabetic patients who received bilateral PRP using the argon laser. The aims of our investigation were to (1) determine the prevalence of failure to attain driving standards in this patient group and (2) determine the contribution of field loss solely attributable to treatment.

\section{METHOD}

One hundred consecutive patients with proliferative diabetic retinopathy attending the Diabetic Clinic, Glasgow Eye Infirmary, were assessed in accordance with the DVLA guidelines. They had visual acuity of at least hand movements in the poorer eye, and as

Eye (1995) 9, 517-525 ㅇ 1995 Royal College of Ophthalmologists 
such were deemed binocular with respect to DVLA field testing. Visual acuity was assessed using the Snellen chart with appropriate spectacle correction.

Visual fields were assessed using the Esterman Visual Field Test (EVFT), which is now available on the Humphrey visual field analyser. ${ }^{15-18}$ This test is recognised by the International Perimetric Society and is recommended by the American Medical Association (AMA) in its guides to visual impairment. ${ }^{19}$ It has recently been adopted by the Visual Standards Sub-Committee of the Royal College of Ophthalmologists who make recommendations to the DVLA and to the Minister of Transport ${ }^{20}$ of the United Kingdom.

The EVFT provides an index which may be as clinically useful for visual fields as the Snellen test is for visual acuity assessment. It consists of a grid of 100 units for monocular and 120 units for binocular tests, which increase in area from centre to periphery (Fig. 1). This produces a relative value scale based on function, with greater weighting in areas most useful to the patient: higher in the centre than the periphery; higher in the inferior than the superior field; and higher near the horizontal meridian than in the rest of the periphery. Each unit equates to one test point and is given a value of 1 (i.e. $100 \times 1=$ $100 \%$ ) in the monocular field, and 0.83 (i.e. $120 \times$ $0.83=100 \%$ ) in the binocular field. The field analyser yields the functional score automatically as a percentage of units seen and displays this on the printout.

In the binocular mode the video eye monitor is aligned on the bridge of the nose enabling central head positioning. Stability of fixation was monitored indirectly by operator observation since the automatic controls of fixation using the blind spot could not be employed.

If poor fixation occurs during the test it should be repeated. If the patient needs spectacles whilst driving the test is performed using the patient's

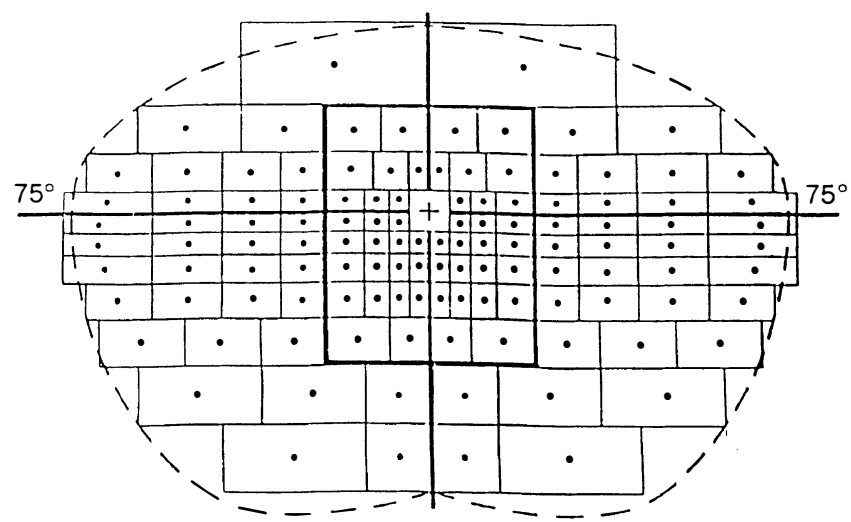

Fig. 1. Binocular Esterman grid overlay. Each of the 120 rectangular areas is given an equal weighting of 0.83 to give a total score of $100 \%$ for a field with no recorded defects. The increasing size of these areas with distance from fixation is related to their functional value. own correction and not trial lenses. If the results are equivocal, the test is performed without the patient's correction to assess whether the frame is obstructing the field of vision.

The EVFT has previously been compared with Goldmann perimetry currently accepted by the DVLA. ${ }^{21}$ No significant variation occurred between field test scores using each form of perimetry. As 86 points of the EVFT lie within the DVLA minimum field for safe driving (corresponding to an EVFT score of $86 \times 0.83=71 \%, \pm 3 \%$ variation of performance), the following guidelines relating to DVLA interpretation of EVFT scores (\%) were recommended: $>74 \%$, pass; $68-74 \%$, borderline pass, reassess using another recognised field test; $<68 \%$, fail.

We also investigated whether any relationship existed between the extent of laser therapy administered and the visual field loss recorded for the first 25 patients with documentation of all previous laser therapy.

\section{RESULTS}

Thirty per cent of patients failed to reach the visual standards required for a UK driving licence. Three groups were identified:

1. The first group $(n=4)$ consisted of those who failed to attain the VA recommendations but met field recommendations. All patients had previously undergone limited PRP consisting of burns of $200 \mu \mathrm{m}$ only to either eye. Vision was compromised due to bilateral macular ischaemia in 3 patients and due to bilateral macular traction in 1 patient.

2. The second group $(n=9)$ consisted of those who failed to attain field recommendations but met VA recommendations. Xenon arc had been given to 2 patients in addition to laser, producing severe field constriction. Of the 7 patients treated with laser alone, 5 were clear fails and 2 were borderline fails. The majority of clear fail laser patients, excluding 1, had received extensive laser photocoagulation of burns greater in size than $200 \mu \mathrm{m}$ to each eye. All borderline fail laser patients had received extensive laser photocoagulation with $200 \mu \mathrm{m}$ burns only to each eye.

3 . The third group $(n=17)$ consisted of those who failed to attain both VA and field requirements. All patients had received a mixture of burns with many greater than $200 \mu \mathrm{m}$ to each eye. Ten patients had bilateral macular ischaemia in addition to proliferative retinopathy. The remaining 7 patients had unilateral retinal detachment, 2 of whom subsequently received surgery which was unsuccessful. These detachments produced severely restricted visual fields and residual acuities of hand movements. Each patient's 


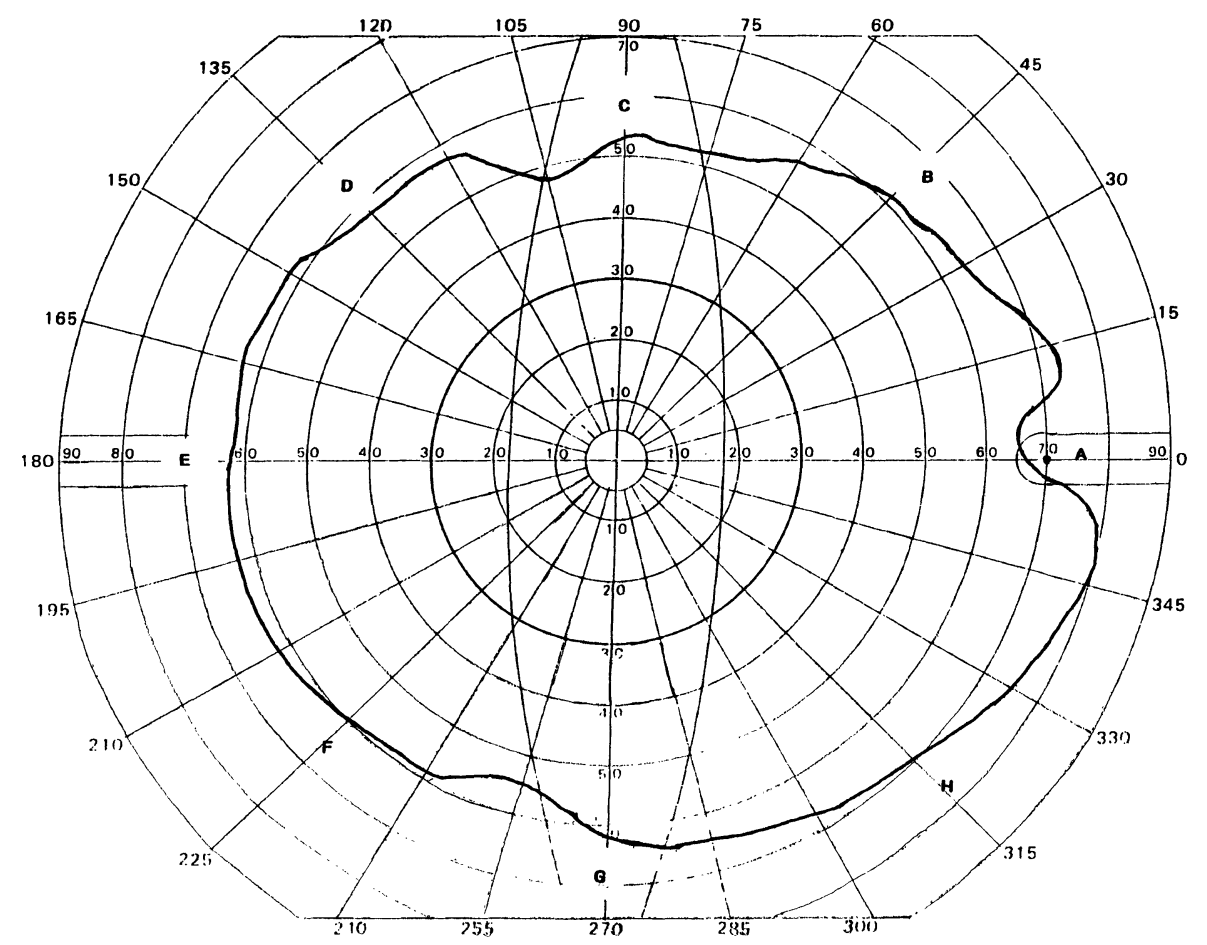

(a)

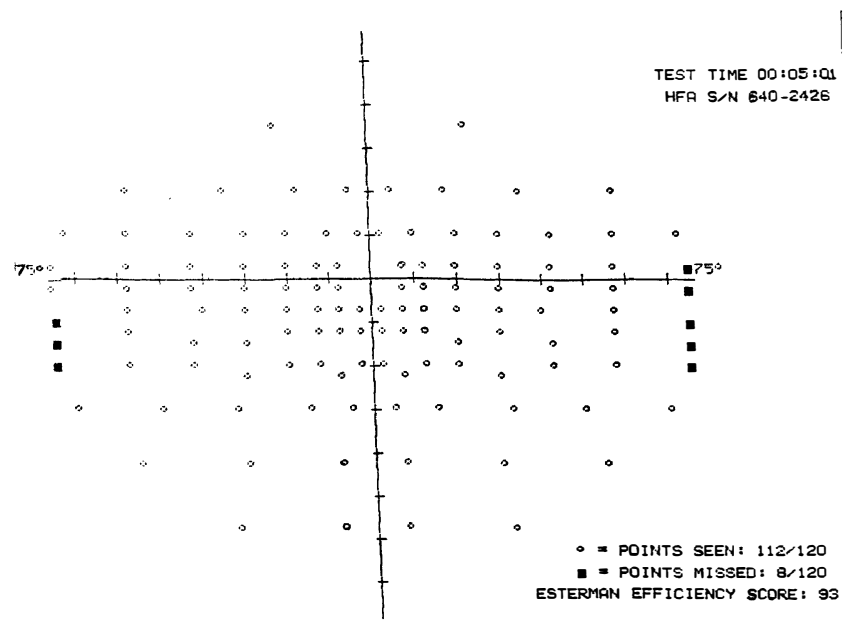

(b)

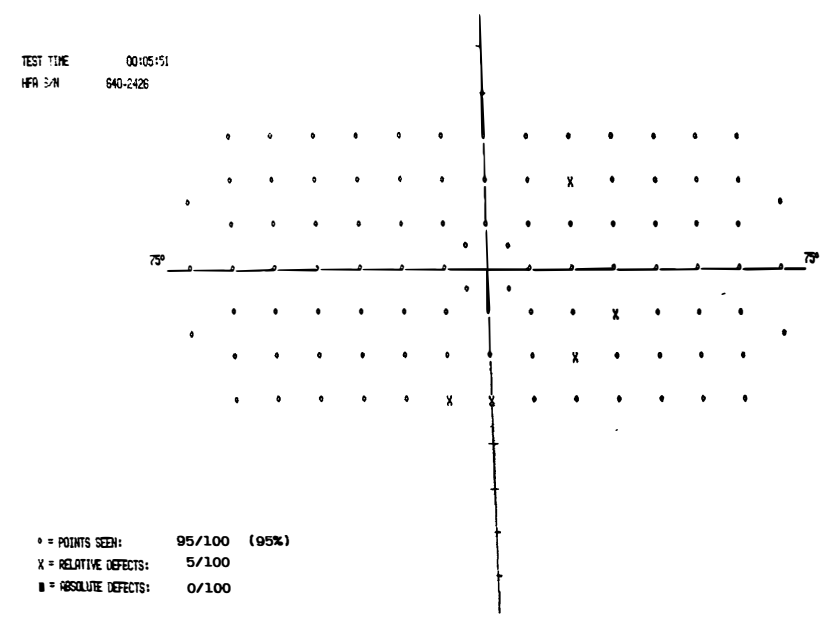

(c)

Fig. 2. Example of visual field plots using three forms of perimetry from the same patient. All of these driving fields clearly pass DVLA recommendations but the patient failed the visual acuity standard. (a) The Goldmann kinetic visual field plot, stimulus III4e. The scores using this apparatus were: Esterman overlay $110 \times 0.83=92 \%$, Meridian method, along the principal meridians $A-H$ indicated, $495 / 500=99 \%$. (b) The Humphrey Esterman static visual field plot, stimulus III4e. The score using this method was $112 \times 0.83=93 \%$. (c) The custom static visual field plot used with the Humphrey Visual Field Analyser, stimulus III4e. The score using this method was 95/100 =95\%. 


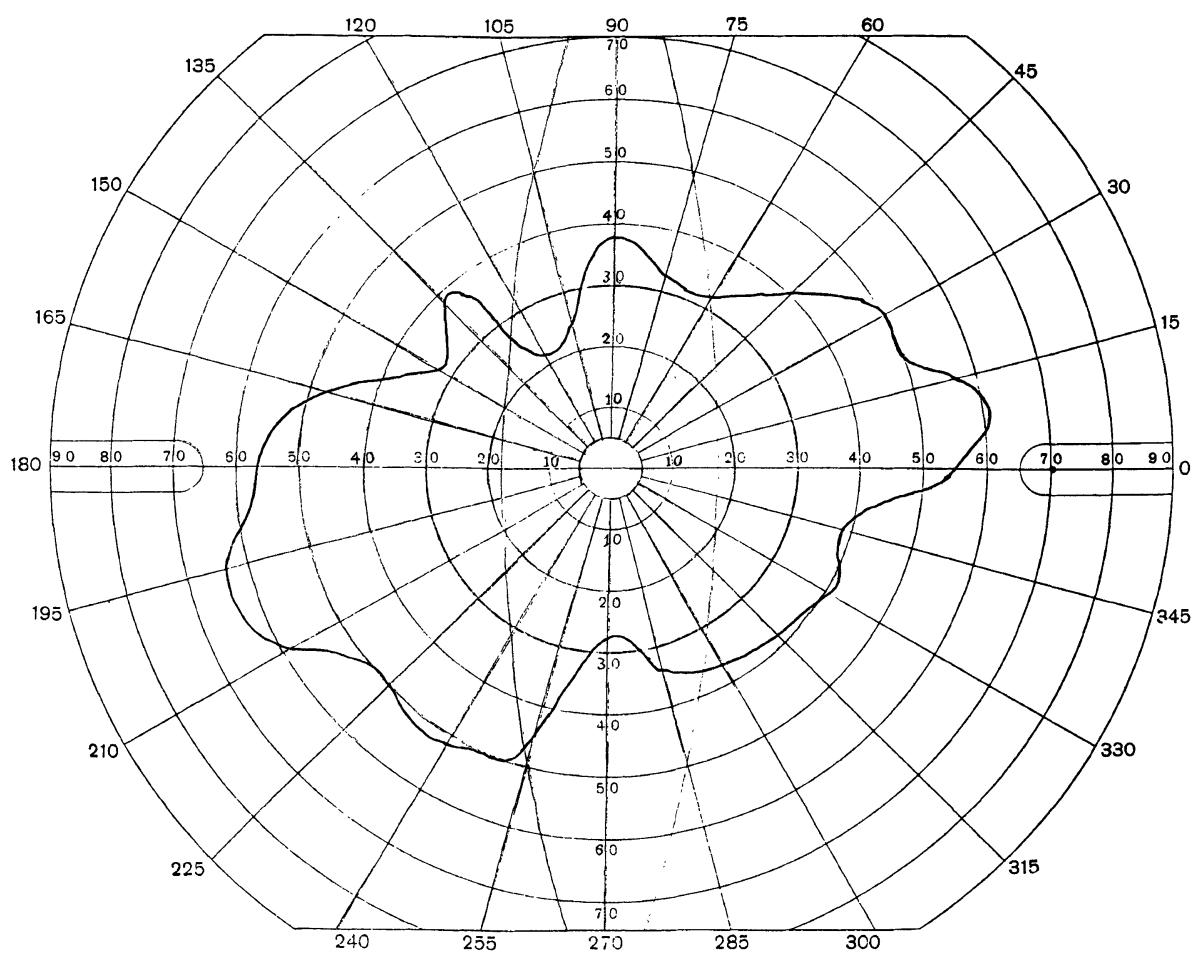

(a)

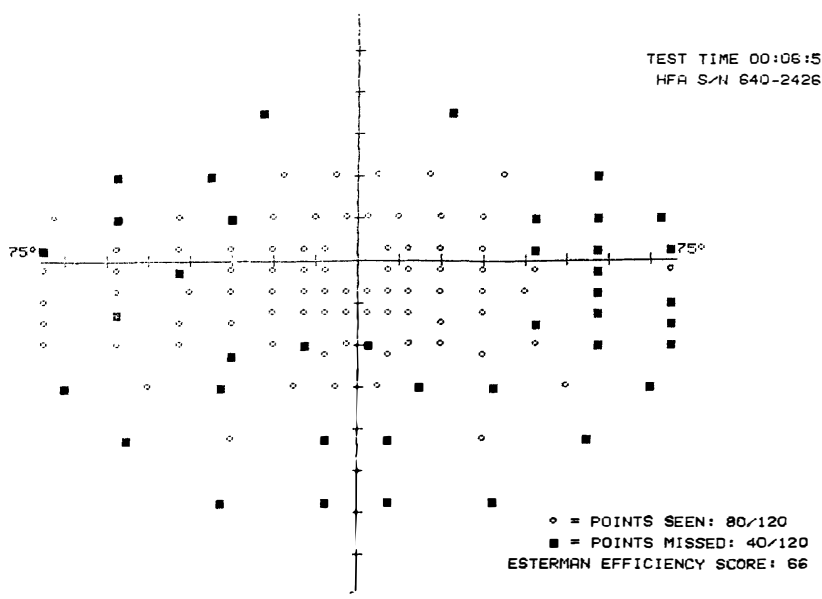

(b)

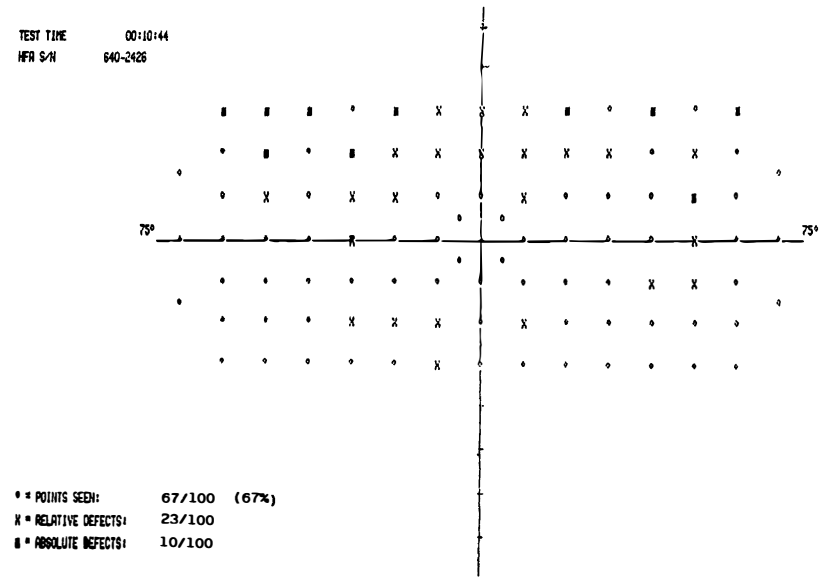

(c)

Fig. 3. Example of visual field plots using three forms of perimetry from the same patient. All these driving fields were borderline fails with respect to DVLA recommendations but the patient passed the visual acuity standard. (a) The Goldmann kinetic visual field plot, stimulus III4e. The scores using this apparatus were: Esterman overlay $84 \times 0.83=70 \%$, Meridian method 345/500 $=70 \%$. (b) The Humphrey Esterman static visual field plot, stimulus III4e. The score using this method was $80 \times 0.83=66 \%$. (c) The custom static visual field plot used with the Humphrey Visual Field Analyser, stimulus III4e. The score using this method was $67 / 100=67 \%$. 


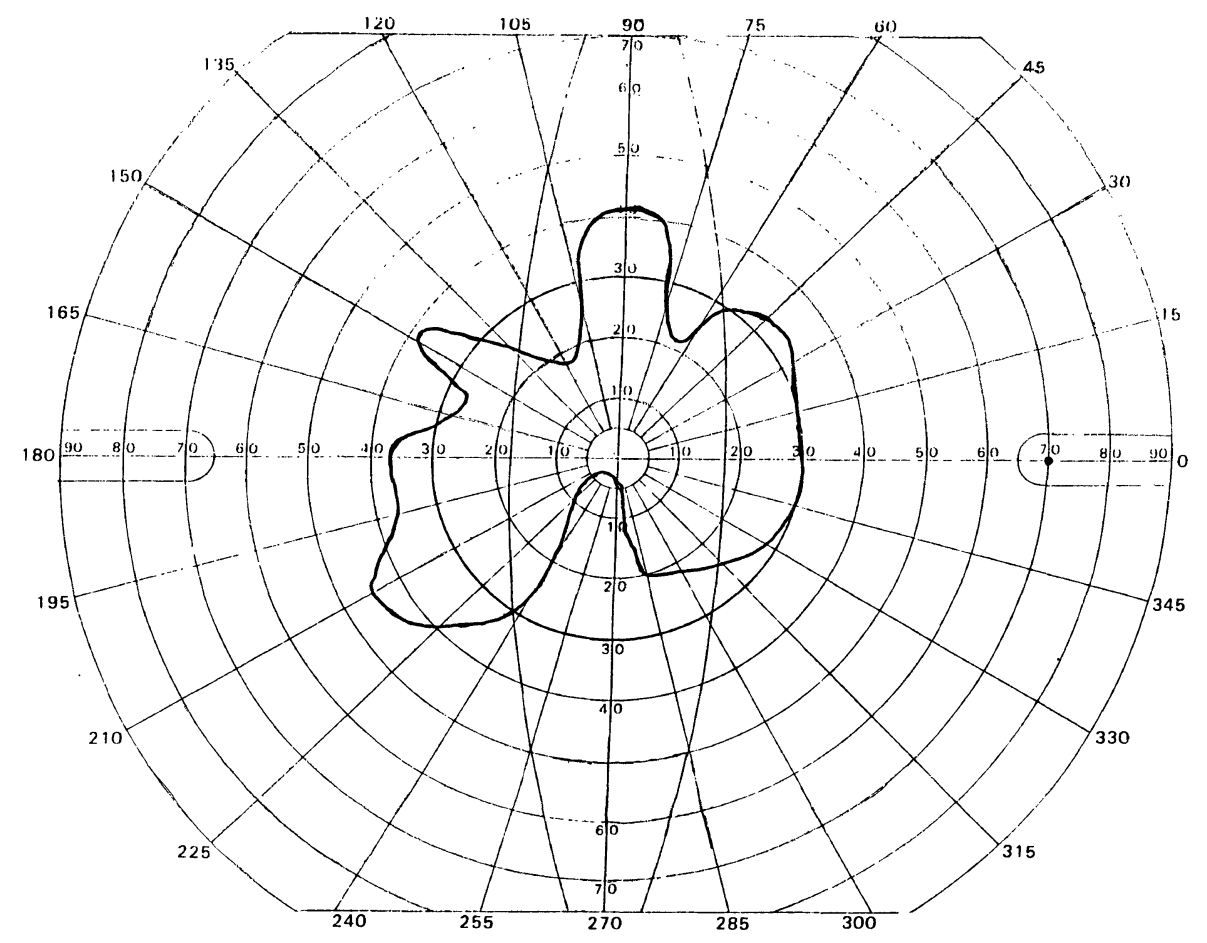

(a)

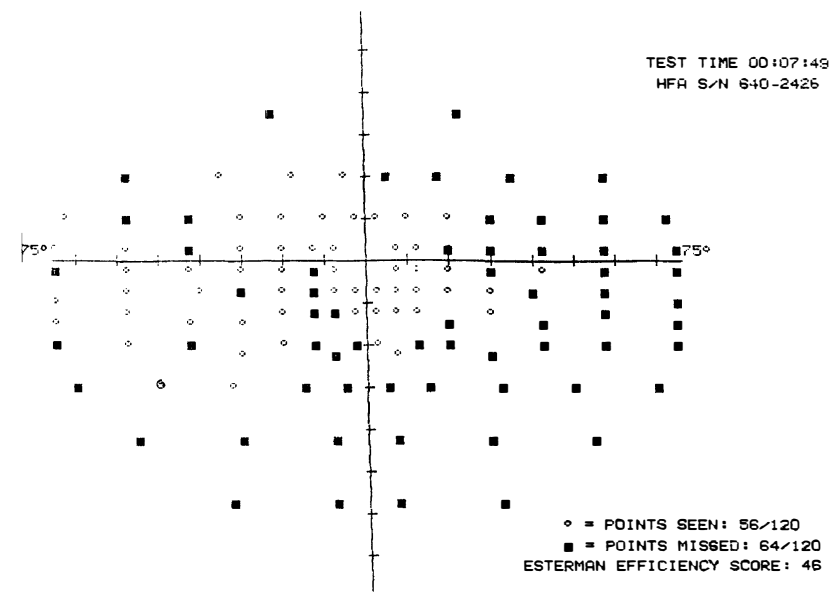

(b)

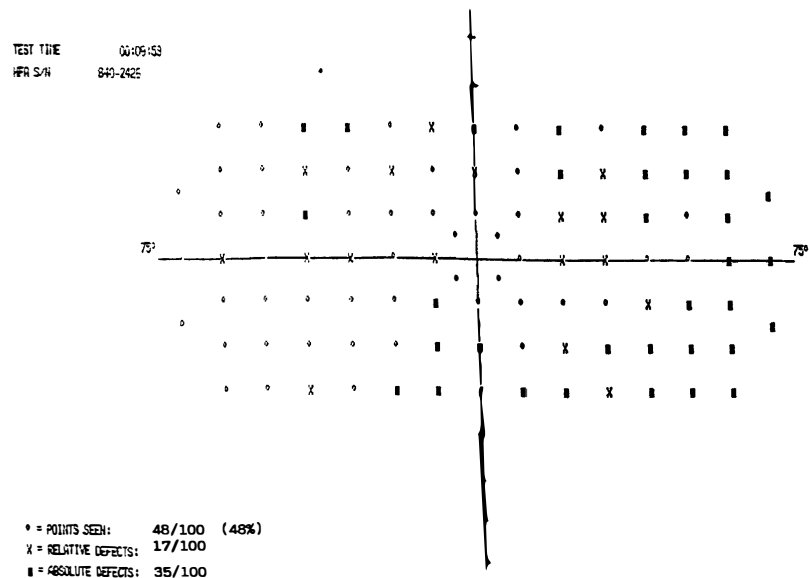

(c)

Fig. 4. Example of visual field plots using three forms of perimetry from the same patient. All of these driving fields clearly failed DVLA recommendations and the patient failed the visual acuity standard. (a) The Goldmann kinetic visual field plot, stimulus III4e. The scores using this apparatus were: Esterman overlay $53 \times 0.83=46 \%$, Meridian method $230 / 500=46 \%$. (b) The Humphrey Esterman static visual field plot, stimulus III4e. The score using this method was $56 \times 0.83=46 \%$. (c) The custom static visual field plot used with the Humphrey Visual Field Analyser, stimulus III4e. The score using this method was $48 / 100=48 \%$. 
fellow 'better' eye had received burns greater than $200 \mu \mathrm{m}$ for proliferative retinopathy. The field loss induced by the laser in combination with the restricted field of their 'poor' eye produced a binocular field less than the required minimum in all patients. Furthermore the visual acuity of each patient's 'better' eye was also compromised, producing a binocular visual acuity less than the required minimum due to recurrent vitreous haemorrhage (3), macular fibrovascular membrane (1), macular ischaemia (1), cataract (1) and rubeotic glaucoma (1).

One patient from each group, as outlined above, had two field plots performed in addition to the EVFT (Figs. 2-4). These comprised a binocular custom test (Humphrey static) and a binocular Goldmann (Topcon Kinetic).

The results were compared, as in a previous study, ${ }^{21}$ by:

1. Using a customised Esterman grid overlay for the Goldmann recording chart to obtain Esterman visual field efficiency scores. In this method the sum of dots on the overlay lying inside the isopter was multiplied by 0.83 to yield a manual score. Any dots lying within a scotoma are subtracted from the final score.

2. Using the meridian method adopted by the AMA $^{19}$ for the Goldmann recording chart to obtain traditional visual field efficiency scores. In this method the angular positions of the isopter as it crosses the eight principal meridians are added together and divided by 500 to yield a manual score. Any scotoma's width is subtracted from the

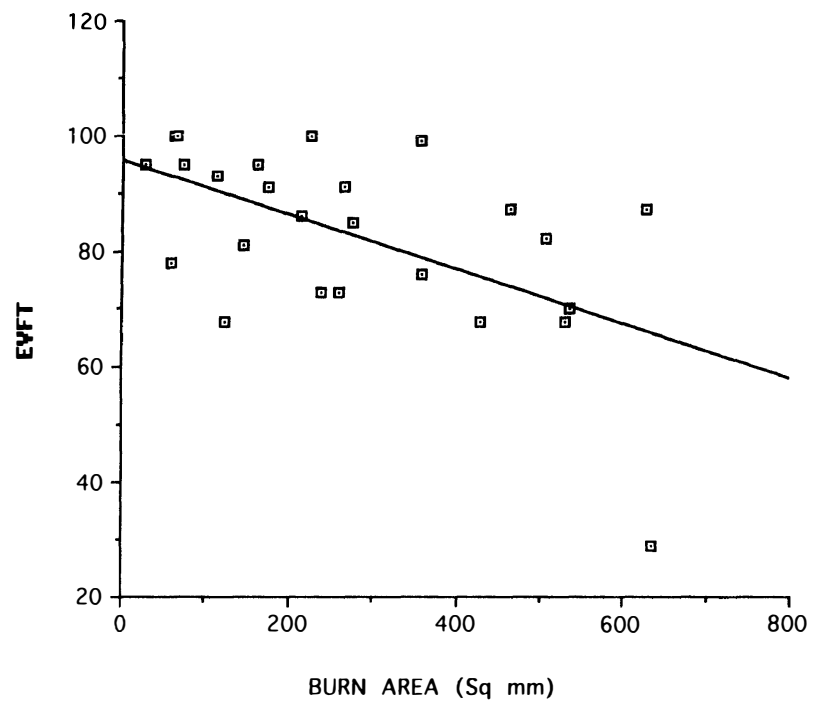

Fig. 5. Scattergram of total burn area (as calculated from patient's treatment details) versus field loss. EVFT score appears to decline slightly with total burn area for the first 25 consecutive patients on which the computations were made, but the correlation is weak $(\mathrm{y}=95.720-4.6579 e-$ $\left.2 \mathrm{x} ; \mathrm{r}^{2}=0.317\right)$. values of the meridians crossing through it before division.

One-way analysis of variance revealed no significant variation between field plots $(p=0.001)$ for each patient, in all three groups.

A scattergram between estimated total burn area and the EVFT score $(n=25)$ was produced (Fig. 5). A least squares regression showed poor correlation between these two parameters $\left(r^{2}=0.32\right)$.

\section{DISCUSSION}

Although $26 \%$ of patients treated with argon laser PRP failed to reach the visual field standard required for a driving licence, only $19 \%$ of failures were attributable solely to the treatment. The remaining $7 \%$ failed as a result of additional complications.

Previous studies reveal a large variation (20$80 \%)^{22,23}$ in DVLA field test failure following PRP treatment for proliferative diabetic retinopathy. Our results are at the lower end of this range. In addition, empirically one might expect a close relationship between the total burn area and the degree of visual field loss.

There are several relevant factors which may offer some possible explanations for these findings.

\section{Burn Size and Distribution}

A standard PRP initial treatment protocol has been adopted at the diabetic eye clinic, Glasgow Eye Infirmary, over the last 4 years. This consists of applying at least 3000 argon laser burns at the $200 \mu \mathrm{m}$ setting. This administration of smaller photocoagulation burns than previously employed has recently been shown to reduce the likelihood of failing to meet DVLA recommendations for visual field standards. ${ }^{22}$ If additional treatment is required, areas already treated should be avoided to prevent additional nerve fibre layer damage and compromise of receptive fields. ${ }^{24}$ Furthermore, argon laser treatment should not be undertaken following fluorescein angiopathy as this can also cause damage to the nerve fibre layer. ${ }^{25}$

\section{Mode of Delivery}

The majority of patients in our study had PRP treatment delivered to each eye by a Mainster contact lens or occasionally by a 90 dioptre ophthalmoscopic Volk lens. The Mainster contact lens produces a $58 \%$ increase in the working field of view and a $3 \%$ increase in magnification is achieved in comparison with the Goldmann contact lens. In theory this produces a retinal spot size $5 \%$ larger than the calibrated photocoagulator spot size setting. ${ }^{26}$ The $4 \mathrm{~mm}$ target (III4e) on the Goldmann perimeter subtends $0.7^{\circ}$ at the nodal point of the eye producing a $210 \mu \mathrm{m}$ diameter spot on the retina of an eye of average axial length. ${ }^{22}$ Thus the enlargement 


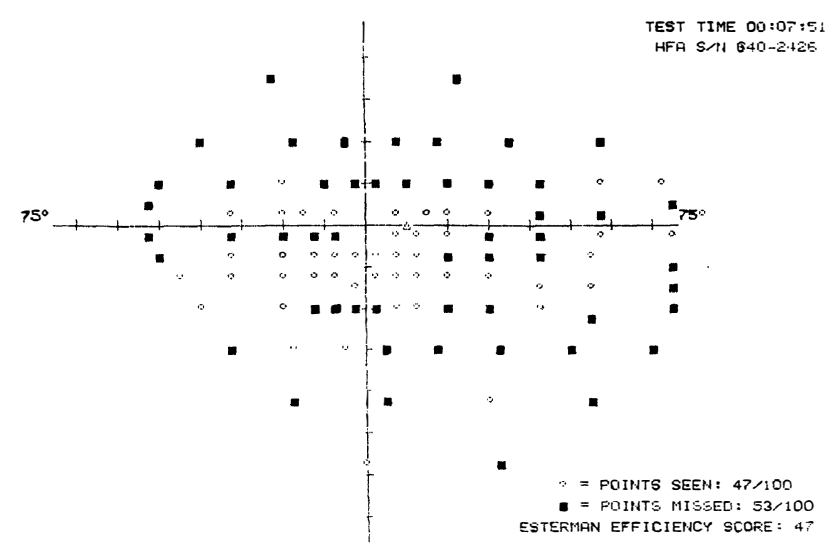

(a)

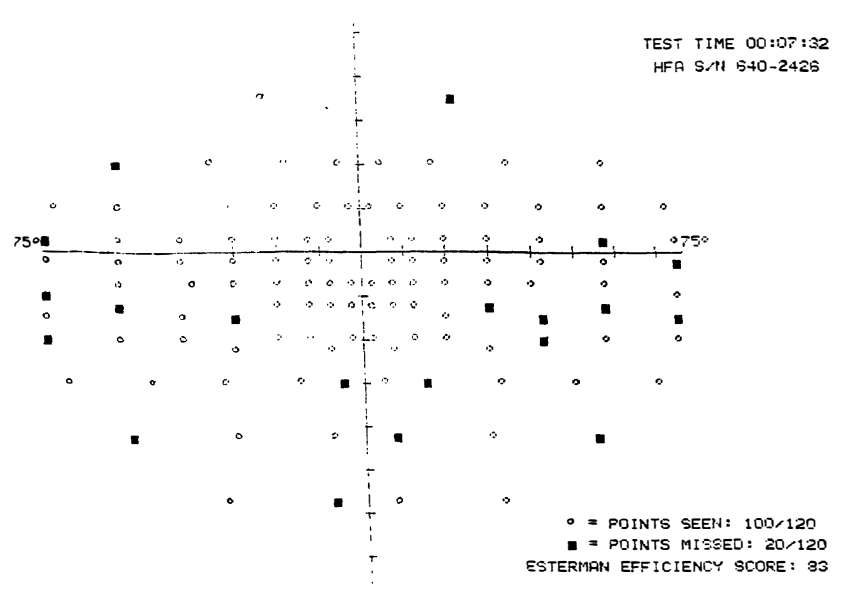

(c)

is unlikely to be significant for non-confluent burns since it is smaller than the angular subtense of the DVLA target dimensions. Perhaps a more significant factor is that of variable expansion of laser burns after application. ${ }^{27}$

\section{Persistent Non-clearing Vitreous Haemorrhage}

Three patients had undergone vitrectomy in one eye for long term non-clearing vitreous haemorrhage. They all retained central vision within the DVLA guidelines but they failed to meet visual field recommendations due to a combination of laser treatment and ischaemic retinopathy. The Diabetic Retinopathy Vitrectomy Study indicated that earlier vitrectomy in patients with aggressive proliferative disease may provide better anatomical results and be associated with maintenance of visual field as well as better vision. $^{28}$

\section{Perimetry Analysis and Binocular Enhancement}

The EVFT is compatible with Goldmann perimetry and allows the application of the DVLA standard to be performed quickly under repeatable conditions,

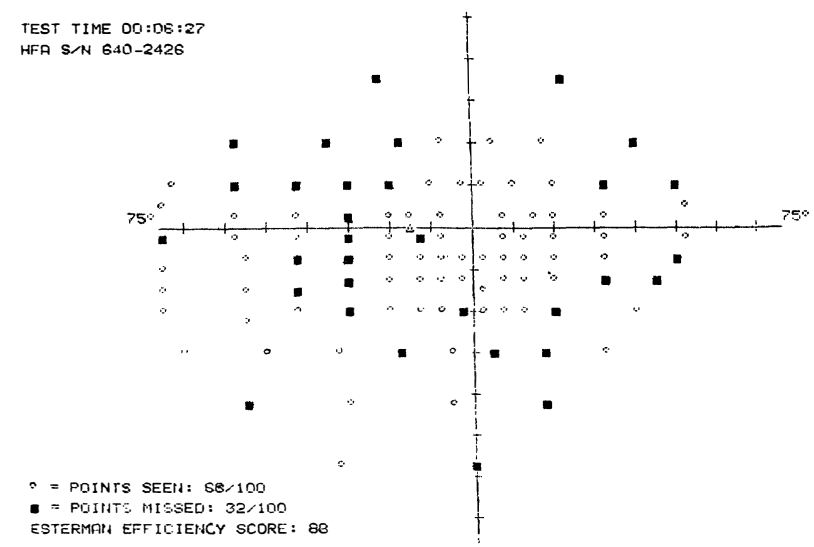

(b)

Fig. 6. Example of three Esterman visual field plots for the same patient. Binocular peripheral enhancement can be seen to occur where the two monocular nasal fields overlap. Areas of field loss in one eye can be compensated for by functional areas of corresponding field in the fellow eye. (a) The Humphrey Esterman static visual field plot, stimulus III 4 e, right eye. The score using this method was $47 \times 1.00$ $=47 \%$. (b) The Humphrey Esterman static visual field plot, stimulus III4e, left eye. The score using this method was 68 $\times 1.00=68 \%$. (c) The Humphrey Esterman static visual field plot, stimulus III4e, both eyes. The score using this method was $100 \times 0.83=83 \%$.

using an automated perimeter. ${ }^{21}$ The EVFT adopts the original Goldmann III4e target notation. However, it varies from Goldmann perimetry with regard to strategy, target luminance and distribution.

Strategy: the EVFT uses static stimuli; Goldmann perimetry usually employs kinetic stimuli.

Target luminance: the EVFT target is very bright (3150 asb) in relation to the Goldmann target (1000 asb). This produces a $0.5 \mathrm{log}$ unit difference in targetto-background luminance. ${ }^{14}$

Distribution: the EVFT is not evenly distributed, being weighted in more functional areas. Interestingly, the Neurologists Advisory Panel accords greater importance to inferior field defects whereas the Visual Standards Sub-Committee of the Royal College of Ophthalmologists is of the opinion that all severe quadrantanopic defects are hazardous for driving. ${ }^{20}$

Current recommendations state that homonymous scotomata which come close to fixation, whether hemianopic or quadrantanopic, would not be accepted as safe for driving. The question arises as 
to how closely these scotomata can approach fixation before constituting a fail. This allows a small degree of flexibility in interpretation; however, isolated static misses due to laser burns, ischaemic areas or poor attention are currently not included within the British guidelines.

An additional factor to consider is the effect of binocular peripheral enhancement. Enhancement occurs in areas where the two monocular nasal fields overlap. This greater sensitivity can increase the binocular Esterman score up to $20 \%$ above that predicted by merging both monocular fields ${ }^{29}$ (Fig. 6). Furthermore, binocular assessment has the advantage of more closely approximating the reallife situation of the patient.

Finally, we are advised by the Visual Standards Sub-Committee (G. Munton, recent Chairman, personal communication) that the recommended standard should not be applied in an unduly restrictive manner. Loss of independence and the important civil liberty of the right to drive should be weighed against the lack of correlation between defects in vision and accident rate, with the exception of data pertaining to side collision. ${ }^{30}$

We conclude that the use of PRP in the treatment of proliferative diabetic retinopathy can be undertaken with the knowledge that the minimum required visual field for driving is maintained in the majority of cases (approximately $80 \%$ ). However, our data suggest that the residual binocular driving field cannot be anticipated from the amount of laser therapy administered and the optimum strategies which combine effective treatment with a wide functional visual field have yet to be established.

The authors are grateful to Gordon Dutton for his review of the manuscript. S.W.M. was supported by a Glasgow Caledonian University Research Scholarship, which is gratefully acknowledged.

Key words: Diabetes, Driving, Esterman test, Photocoagulation, Retinopathy, Visual fields.

\section{REFERENCES}

1. Diabetic Retinopathy Research Group. Photocoagulation treatment of proliferative diabetic retinopathy: the second report of diabetic retinopathy study findings. Ophthalmology 1978;85:82-106.

2. Huamonte PE, Penman GA, Goldberg MF, et al. Immediate fundus complications after retinal scatter photocoagulation. I. Clinical picture and pathogenesis. Ophthalmic Surg 1976;7:88-99.

3. Doft BH, Blackenship GW. Single versus multiple treatment sessions of argon laser pan-retinal photocoagulation for proliferative diabetic retinopathy. Ophthalmology 1992;89:772-9.

4. Lewis $\mathrm{H}$, Schachat AP, Haimann $\mathrm{MH}$, et al. Choroidal neovascularisation after laser photocoagulation for diabetic macular edema. Ophthalmology 1990;97:50311.

5. Ferris FL, Podgor MJ, Davis MD. Macular edema in
Diabetic Retinopathy Study patients: the twelfth report of the Diabetic Retinopathy Study Research Group. Ophthalmology 1987;94:754-60.

6. Swartz M, Apple DJ, Creel D. Sudden severe visual loss associated with peri-papillary burns during panretinal argon photocoagulation. $\mathrm{Br} \mathrm{J}$ Ophthalmol 1983;67:517-9.

7. McCanna P, Chandra SR, Stevens TS, et al. Argon laser-induced cataract as a complication of retinal photocoagulation. Arch Ophthalmol 1982;100:1071-3.

8. Sieberm V, Alexandrides E, Feng W. Function of the diabetic retina after pan-retinal argon laser coagulation. Graefes Arch Clin Exp Ophthalmol 1987;225:385-90.

9. Trick GL, Burde RM, Gordon MO, Santiago JV, Kilo C. The relationship between hue discrimination and contrast sensitivity deficits in patients with diabetes mellitus. Ophthalmology 1989;95:693-8.

10. Mackie SW, Barrie T, Elliott DB, MacCuish AC, Walsh G. Glare disability in diabetic patients before and after panretinal photocoagulation. Invest Ophthalmol Vis Sci 1994;35:1593.

11. Khosala PK, Talwar D, Tewari HK. Contrast sensitivity changes in background diabetic retinopathy. Can J Ophthalmol 1991;26:7-11.

12. Road Traffic Act 1988. Provision of information etc. relating to disabilities. London: HMSO.

13. Drasdo N, Haggerty CM. A comparison of the British number plate and Snellen vision tests for car drivers. Ophthalmol Physiol Opt 1981;1:39-54.

14. Form CLE 1060. DVLC Drivers Medical Branch, revised September 1992

15. Esterman B. Functional scoring of the binocular field. Ophthalmology 1982;89:1226-34.

16. Esterman B. Grid for scoring visual fields. II. Perimeter. Arch Ophthalmol 1968;79:400-6.

17. Esterman B, Blanche E, Wallach M, Bonelli A. Computerised scoring of the functional field: preliminary report. Doc Ophthalmol Proc Ser 1985;42:333-9.

18. Choy ES, Mills RP, Drance SM. Automated Esterman testing of disability in glaucoma. In: Seventh International Visual Field Symposium. Amsterdam, 1986.

19. American Medical Association. Guides to the evaluation of permanent impairment, 3rd ed. Chicago: American Medical Association, 1988.

20. Redefinition of the minimal field of vision for safe driving: guidelines. London: Royal College of Ophthalmologists, 1990.

21. Mackie SW, Barrie T, McClure E. Walsh G. Use of the Esterman visual field test in diabetics following panretinal photocoagulation with respect to DVLA recommendations. In: Vision in vehicles IV. Amsterdam: North-Holland/Elsevier, 1995 (in press).

22. Hulbert MFG, Vernon SA. Passing the DVLC field regulations following pan-retinal photocoagulation in diabetics. Eye 1992;6:456-60.

23. Williamson TH, George N, Flanagan DW, Norris V, Blamires T. Driving standards: visual fields in diabetic patients after pan-retinal photocoagulation. In: Vision in vehicles III. Amsterdam: North-Holland/Elsevier, 1991:265-72.

24. Frank RN. Visual fields and electroretinography following extensive photocoagulation. Arch Ophthalmol 1975;93:591-8.

25. Parks S, Aiken D, Keating D, Dutton GN. Argon-laser photocoagulation of fluorescein stained retina, an unrecognised hazard? Br J Ophthalmol 1994;78:466-7.

26. Mainster MA, Crossman JL, Erickson PJ, Heathcot 
GL. Retinal laser lenses: magnification, spot size and field of view. Br J Ophthalmol 1990;74:177-9.

27. Schatz H. et al. Progressive enlargement of laser scars following grid laser photocoagulation for diffuse diabetic macular oedema. Arch Ophthalmol 1991;109:1549-51.

28. Diabetic Retinopathy Vitrectomy Study Research Group. Report no. 2. Early vitrectomy for severe vitreous haemorrhage in diabetic retinopathy: two-year results of a randomized trial. Arch Ophthalmol 1985;103:1644-52.

29. Mills RP, Drance SM. Esterman disability rating in severe glaucoma. Ophthalmology 1986;93:371-8.

30. Council FM, Allen JA. A study of the visual fields of North Carolina drivers and their relationship to accidents. Highway Safety Research Centre, University of North Carolina, 1974. 